\title{
Self-perceived fatigue in adolescents in relation to body composition and physical outcomes
}

\author{
Stijn Vantieghem ${ }^{1}$, Ivan Bautmans ${ }^{2}$, Jonathan Tresignie ${ }^{1}$ and Steven Provyn ${ }^{1}$
}

BACKGROUND: Increased self-perceived fatigue (SpF) has already been identified in chronic conditions such as obesity, but it is also a growing problem in school-attending adolescents ( $\pm 25 \%)$. This study tried to link body composition to $\mathrm{SpF}$ and physical activity/performance. Additionally, indicators for fatigue were determined.

METHODS: A total of 452 adolescents were recruited. Body composition was measured and physical activity, physical performance, and SpF were assessed. Based on the total SpF (Multidimensional Fatigue Inventory) outcomes, three groups were created: low fatigue (LF) medium fatigue (MF) and high fatigue (HF).

RESULTS: Fat was significantly lower in the LF group compared with MF $(P<0,05)$ and HF $(P<0.01)$. Grip endurance was increased in LF $(P<0.05)$ and MF $(P<0.01)$ compared with HF; similar results were found with the Cooper test. Sport Index was increased in LF compared with MF and HF $(P<0.01)$. Fat and physical activity were related to fatigue $(P<0.01)$. Decreased fatigue resistance, Sport Index and higher fat percentage increased the chance of being extremely fatigued.

CONCLUSION: This study emphasizes the importance of using fat mass and fat percentage instead of body mass index when screening adolescents. To prevent increased SpF, it is necessary to stimulate youngsters to be physically active and to promote healthy behaviors.

M orphological changes occur during growth, starting from the intrauterine and postnatal period and continue during life. Normally, growth and maturation evolve in harmony. Between 10 and 16 years, hormonal reactivation occurs that induces puberty (1). This bio-chemical process can be disrupted by various factors, such as not only alcohol and malnutrition but also obesity (1-4). During this stage of life, it is important to adapt healthy and avoid unhealthy behaviors. Being physically active is one of these healthy behaviors; various knowledge centers advise adolescents to be active at least $60 \mathrm{~min}$ a day $(5,6)$ and, what is often forgotten, to decrease recreational screen time (sedentary behavior) to no $>2 \mathrm{~h}$ a day (7). Adherence to these recommendations results in improved weight management and insulin sensitivity and decreased hypertension and blood pressure $(8,9)$.

Physical education (PE) teachers seem to have an important assignment in encouraging adolescents to be more active, knowing that physical activity in youth is significantly lower than the recommended guidelines $(10,11)$ and often limited to an average of only $2 \mathrm{~h}$ of PE classes a week. A longitudinal study found that increased fat mass and body mass index (BMI) compromises physical fitness in adolescents (12), meaning that being physically active is an important determinant for the weight status of youngsters (13). Body composition parameters are already well described and related to physical health. However, increasing numbers of overweighed and obese children in Europe remain very disturbing. In Belgium, these conditions are progressing rapidly: the rate for overweight increased between 1997 and 2008 from $41 \%$ to $47 \%$ and for obesity from $11 \%$ to $14 \%$ (14). To prevent the development of future obesity-related morbidities (such as cardiovascular pathology, diabetes, depression, dyslipidemia, and so on), it is important to monitor overweight and obesity in children and adolescents $(15,16)$.

Beside these physiological concerns, more attention is currently given to the psychological aspects of the individual such as depression, quality of life, and fatigue $(17,18)$. In literature, self-perceived fatigue $(\mathrm{SpF})$ is often related to (chronic) illnesses, such as cancer, fibromyalgia, rheumatology, obesity, etc (19-23). Nevertheless, data on schoolattending adolescents report $>25 \%$ severe $\mathrm{SpF}$ at least once a week (24). Reducing this SpF in secondary high schools can be of importance because of its negative impact on cognitive function (25) and possibly on school results.

Previous literature demonstrated that depression and reduced quality of life were linked to increased fat percentage (17), and improved quality of life was achieved after a weight loss program with increased physical activity (18). To our knowledge, no study investigated the inter-relations between body composition, muscular fatigue, physical activity/performance, and SpF in normal weight school-attending adolescents. The main aim of this study was to determine whether body composition parameters (fat in particular), muscular

\footnotetext{
${ }^{1}$ Anatomical Research and Clinical Studies, Vrije Universiteit Brussel (VUB), Brussels, Belgium; ${ }^{2}$ Frailty in Ageing, Vrije Universiteit Brussel (VUB), Brussels, Belgium. Correspondence: S Vantieghem (stijn.vantieghem@vub.be)

Received 24 May 2017; accepted 16 October 2017; advance online publication 15 November 2017. doi:10.1038/pr.2017.274
} 
fatigue, and being physically active were predictors for SpF. Second, this study tried to find relations between body composition, physical performance, and physical activity.

\section{METHODS}

\section{Recruitment and participants}

All pupils $(N=452)$ were recruited at PIVA high school (Antwerp, Belgium) with a technical and professional curriculum. Pupils were aged between 12 and 20 years with a 66/34 boy/girl ratio. Data were collected by experienced PE teachers in the second week after the summer holidays as a yearly routine. Pupils could decline to participate by indicating this to their PE teacher. Only one pupil declined to participate. The data were used retrospectively and approved by the Academic Bio-Ethical Committee Human Research Brussels (IRB B200-2015-053).

\section{Anthropometric and body composition measurements}

Standing height was measured with a stadiometer (seca 217, SECA, Hamburg, Germany) to the nearest $0.1 \mathrm{~cm}$ and both body weight and body composition (fat mass, fat percentage, fat-free mass, and muscle mass) were determined with a class III Bioelectrical Impedance Analysis device, Tanita MC-180MA to the nearest $0.1 \mathrm{~kg}$ (Tanita, Tokyo, Japan) following a standardized protocol (26). BMI was calculated as weight/height ${ }^{2}\left(\mathrm{~kg} / \mathrm{m}^{2}\right)$.

\section{Physical activity}

Physical activity was scored using the validated Baecke questionnaire (27). School/work activities, sport activities, and leisure time were scored to asses three subscales: School/Work Index (SWI), Sport Index (SI), and Leisure Time Index (LTI). For each domain, a dimensional score was obtained.

\section{Self-perceived fatigue}

Self-perceived fatigue was assessed using the Dutch (28) version of the Multidimensional Fatigue Inventory (MFI-20). The MFI-20 covers five domains of fatigue: general fatigue, physical fatigue, mental fatigue, reduced motivation, and reduced activities. Each subscale includes 4 items with 5-point categories, resulting in a subscale score range of 4-20, with higher scores indicating greater fatigue. Also, a total MFI score (score range from 20 to 100) was calculated by summing up the scores of the 5 subscales. Total fatigue was used to divide the adolescents into three groups, namely, low fatigue (LF), medium fatigue (MF), and high fatigue (HF). The first tertile corresponded with LF, the second tertile with MF, and the highest tertile with HF. Internal consistency of the total MFI-20 score for this population was measured by Cronbach's $\alpha$ (0.87), which indicates a good internal consistency.

\section{Physical performance}

Grip strength (GS) was executed with the dominant hand using a Martin Vigorimeter (KLS Martin Group, Tuttlinger, Germany), a device with a rubber bulb connected to a manometer. GS of the dominant hand was assessed as described previously $(29,30)$. In summary, the shoulder was adducted and neutrally rotated, the elbow flexed at $90^{\circ}$, the forearm in neutral position, and the wrist in slight extension $\left(0-30^{\circ}\right)$. The subject was asked to squeeze the rubber bulb ( 3 sizes available, according to the subject's hand size) as hard as possible for 3 times with a 30-s interval. The highest of three attempts was noted as the maximal GS (GS expressed in KPa). The time (in seconds) during which GS dropped to $50 \%$ of its maximum was recorded as fatigue resistance (FR). Grip work (GW), a parameter reflecting the total effort produced during the FR test, was estimated by multiplying FR (in seconds) with $75 \%$ of the GS (in $\mathrm{KPa}$ ), as described previously (29). This parameter represents the physiological work delivered by the arm muscles during the FR test.

The Cooper test was assessed during PE classes. Pupils were asked to run as far as possible in a 12 -min period. At the start and end of the 12-min period, pupils were asked to, respectively, start and stop running on a whistle signal. Once the distance of a pupil was recorded, (s)he could leave the track. The distance was recorded to the nearest $20 \mathrm{~m}$.

\section{Data analysis}

Data were analyzed using SPSS 23.0 for mac (SPSS, Chicago, IL). Descriptives (mean $\pm S D$ ) were calculated for weight, BMI, and body composition parameters (fat mass, fat percentage, fat free mass, and muscle mass); for physical performance (GS, FR and GW); and for physical activity (School/Work Index, Sport Index, and Leisure Time Index). The population was divided into three groups, based on Total Fatigue outcomes. Differences between the groups (LF, MF, and HF) were calculated with a one-way analysis of variance. If significant F-values were found, post hoc analyses (Bonferroni) were conducted. Correlations (Spearman) were computed between SpF (ordinal) and physical activity (scale) and also between fatigue (self-perceived, ordinal), physical activity (scale), and body composition parameters (scale). Binary logistic regression (method: forward stepwise likelihood ratio) was computed to assess whether the covariates body composition and physical activity/performance can determine the level of fatigue (dependent) in adolescents.

\section{RESULTS}

The adolescents, who were divided into three groups (LF, MF, and HF) based on total SpF fatigue assessed by MFI-20, showed no significant differences for age, body weight, BMI, fat-free mass, and muscle mass (Table 1). Fat mass and fat percentage were significantly lower in LF compared with MF and $\mathrm{HF} \quad(\mathrm{F}=4.67 ; \quad P=0.01$ and $\mathrm{F}=8.11 ; \quad P<0.001$, respectively), with no differences between $\mathrm{MF}$ and $\mathrm{HF}$ $(P>0.05)$ (Table 1).

With regard to physical performance, GS $(\mathrm{F}=1.91$; $P=0.15)$ was equal in all groups, and differences were found

Table 1. Descriptives

\begin{tabular}{|c|c|c|c|c|}
\hline & Low fatigue (a) & Medium fatigue (b) & High fatigue (c) & \\
\hline & Mean $\pm S D$ & Mean $\pm S D$ & Mean $\pm S D$ & \\
\hline Age (years) & $15.22 \pm 1.42$ & $15.33 \pm 1.35$ & $15.28 \pm 1.39$ & NS \\
\hline BMI $\left(\mathrm{kg} / \mathrm{m}^{2}\right)$ & $21.87 \pm 4.06$ & $22.51 \pm 4.75$ & $22.75 \pm 5.17$ & NS \\
\hline Fat mass $(\mathrm{kg})$ & $11.37 \pm 6.30$ & $13.54 \pm 7.88$ & $14.75 \pm 9.62$ & $\mathrm{a}<\mathrm{b}^{*} ; \mathrm{a}<\mathrm{c}^{* *}$ \\
\hline Muscle mass $(\mathrm{kg})$ & $49.50 \pm 9.90$ & $48.61 \pm 9.85$ & $47.48 \pm 9.70$ & NS \\
\hline
\end{tabular}

NS, not significant. ${ }^{*} P<0.05 ;{ }^{* *} P<0.01$. 


\section{Articles | Vantieghem et al.}

Table 2. Physical activity and physical performance in adolescents

\begin{tabular}{|c|c|c|c|c|}
\hline & Low fatigue (a) & Medium fatigue (b) & High fatigue (c) & \\
\hline & mean $\pm S D$ & mean $\pm S D$ & mean $\pm S D$ & \\
\hline Grip strength $(\mathrm{kPa})$ & $98.95 \pm 23.41$ & $100.13 \pm 22.96$ & $94.65 \pm 24.12$ & NS \\
\hline Fatigue Resistance (s) & $42.31 \pm 22.22$ & $45.45 \pm 21.48$ & $36.24 \pm 18.69$ & $a^{*}, b^{* *}>c$ \\
\hline Gripwork $(\mathrm{kPa} \times \mathrm{s})$ & $3218.77 \pm 2059.59$ & $3467.64 \pm 1892.62$ & $2633.98 \pm 1725.12$ & $a^{*}, b^{* *}>c$ \\
\hline Cooper test (m) & $2088.36 \pm 470.41$ & $1899.37 \pm 533.31$ & $1744.37 \pm 426.37$ & $a^{* *}>b^{*}>c$ \\
\hline School/Work Index & $2.70 \pm 0.30$ & $2.77 \pm 0.33$ & $2.701 \pm 0.34$ & NS \\
\hline Sport Index & $3.21 \pm 0.81$ & $2.79 \pm 0.71$ & $2.37 \pm 0.71$ & $a^{* *}>b^{* *}>c$ \\
\hline Leisure Time Index & $3.09 \pm 0.66$ & $3.05 \pm 0.60$ & $2.87 \pm 0.68$ & $a^{* *}, b^{*}>c$ \\
\hline
\end{tabular}

for $\mathrm{FR}(\mathrm{F}=6.25 ; P=0.002)$ and $\mathrm{GW}(\mathrm{F}=6.30 ; P=0.002)$. Data showed significant higher FR and GW in LF and MF compared with HF $(P=0.02$ and $P=0.01$ for FR and GW, respectively; Table 2).

Physical activity showed no significant differences for School/Work Index $(\mathrm{F}=2.10 ; P=0.12)$ between the three groups, although for Sport Index $(\mathrm{F}=46.41 ; P<0.001)$ and Leisure Time Index $(\mathrm{F}=4.89 ; P=0.008)$ significant differences were found (Table 2). Increased Sport Index was found in LF compared with MF and $\mathrm{HF}$ (all $P<0.01$ ). The MF group was more active compared with $\operatorname{HF}(P<0.01)$. Leisure Time Index for LF and MF were higher compared with $\mathrm{HF}(P<0.01$ and $P=0.02$, respectively).

To assess a possible link between body composition, physical activity, physical performance, and $\mathrm{SpF}$, binary logistic regression was performed to assess high or low feelings of fatigue in the population $\left(\chi^{2}=52.30, \mathrm{df}=3\right.$, $N=300, P<0.001$ ). The model explained $42.9 \%$ (Nagelkerke $R^{2}$ ) of the variance in level of fatigue and correctly classified $77.0 \%$ of the cases. FR, Sport Index, and Fat Percentage were selected to assess the level of SpF (LF or HF). Decreased FR, Sport Index, and a higher fat percentage increased the chance of being highly fatigued $\mathrm{SpF}$ group $=-0.023 \mathrm{FR}-1,489$ Sport Index +0.081 fat percentage $+3,663$; removing one of these parameters had a significant impact on the outcome. A total of 16 variables were included to assess HF or LF. Parameters excluded from the equation were: age, sex, BMI, GS, GW, Cooper test, School/Work Index, Leisure Time Index, fat mass, fat-free mass, and muscle mass.

Analyses have shown negative relations between all subscales of MFI-20 and the Sport Index (all $P<0.001$ ). Leisure Time Index was also related to MFI-20 except for subscale Mental Fatigue (Table 3). The School/Work Index was related to subscale General Fatigue $(r=0.13 ; P<0.01)$, Reduced Activity $(r=-0.17 ; \quad P<0.01)$, and Reduced Motivation $(r=-0.12 ; P<0.05)$. No significant relations were found between fat-free mass/muscle mass and MFI-20 or outcomes of the Baecke activity questionnaire. Fat mass and fat percentage were positively related to MFI-20 (Table 4) and to School/Work Index. Fat percentage was negatively related to Sport Index (Table 4).
Table 3. Correlations between self-perceived fatigue and physical activity

\begin{tabular}{lccc}
\hline & $\begin{array}{c}\text { School/Work } \\
\text { Index }\end{array}$ & Sport Index & $\begin{array}{c}\text { Leisure Time } \\
\text { Index }\end{array}$ \\
\hline Total MFI & -0.02 & $-0.48^{* *}$ & $-0.17^{* *}$ \\
General fatigue & $0.13^{* *}$ & $-0.38^{* *}$ & $-0.14^{* *}$ \\
Physical fatigue & 0.05 & $-0.48^{* *}$ & $-0.11^{*}$ \\
Reduced activity & $-0.17^{* *}$ & $-0.43^{* *}$ & $-0.16^{* *}$ \\
Reduced motivation & $-0.12^{*}$ & $-0.26^{* *}$ & $-0.20^{* *}$ \\
Mental fatigue & -0.04 & $-0.18^{* *}$ & -0.03 \\
\hline
\end{tabular}

${ }^{*} P<0.05 ; *{ }^{*} P<0.01$.

Table 4. Correlations between body composition parameters

\begin{tabular}{lcccc}
\hline & $\begin{array}{c}\text { Fat mass } \\
(\mathrm{kg})\end{array}$ & Fat\% & $\begin{array}{c}\text { Fat-free } \\
\text { mass }(\mathrm{kg})\end{array}$ & $\begin{array}{c}\text { Muscle mass } \\
(\mathrm{kg})\end{array}$ \\
\hline Total MFI & $0.18^{* *}$ & $0.25^{* *}$ & -0.11 & -0.11 \\
General fatigue & $0.20^{* *}$ & $0.28^{* *}$ & -0.06 & -0.06 \\
Physical fatigue & $0.35^{* *}$ & $0.43^{* *}$ & -0.06 & -0.06 \\
Reduced activity & 0.08 & $0.11^{*}$ & -0.08 & -0.08 \\
Reduced & -0.02 & -0.01 & -0.08 & -0.08 \\
motivation & & & & \\
Mental fatigue & -0.04 & -0.01 & -0.09 & -0.09 \\
School/Work & $0.13^{*}$ & $0.14^{*}$ & 0.03 & 0.03 \\
Index & & & & 0.10 \\
Sport Index & -0.10 & $-0.13^{*}$ & 0.10 & 0.08 \\
Leisure Time & 0.05 & 0.03 & 0.08 & \\
Index & & & & \\
\hline
\end{tabular}

${ }^{*} P<0.05 ;{ }^{*} P<0.01$.

\section{DISCUSSION}

This study examined $\mathrm{SpF}$ in school-attending adolescents. To our knowledge, it is one of the first studies that tries to link SpF, body composition, physical activity, and physical performance in normal-weight adolescents. Previous literature mentioned that $>25 \%$ of adolescents reported severe SpF at least once a week (24) and that adolescents with chronic illnesses, including cancer, fibromyalgia, rheumatoid arthritis, and obesity $(19,21,23)$, perceived higher fatigue compared 
with healthy peers. Very little data were found on $\mathrm{SpF}$ in normal-weight adolescents.

In this study, a total of 452 adolescents were assessed for physical performance (Cooper test and grip parameters), physical activity, body composition, and SpF. The total score of $\mathrm{SpF}$ was used to subdivide the population into three groups, namely, LF, MF, and HF. No differences in age, weight, and BMI were found between these groups. Comparable results were found in adolescents with a physical disability (31) while Viner et al. (24) reported increased severe fatigue with age. No relation was found between weight status and their perceived fatigue by Maher et al. (31), which was according to our results. Body composition parameters, however (fat mass and fat percentage, in particular) were significantly different between the three SpF groups. Pupils who perceive LF had significantly less fat (expressed in $\mathrm{kg}$ and percentage) compared with MF and HF pupils. These results highlight the negative association between $\mathrm{SpF}$ and fat, but it remains unclear if fat metabolism is (co)responsible for these perceived feelings of fatigue and the direction of causation. Despite the fact that significant differences concerning fat were found between the three groups, BMI values were similar. This also indicates that BMI, which is not an indicator for fat, should be interpreted with caution as a descriptive parameter in adolescents and cannot be replaced with fat mass or fat percentage assessed by bioelectrical impedance analysis. In adults, on the other hand, fat and BMI were both related to fatigue (32). At this time, we cannot explain these opposite results. Fat-free mass and muscle mass did not seem to influence fatigue, in contrast to body fat.

Physical activity and performance were also parameters related to SpF. It was found that pupils with improved muscle fatigue ability (increased FR and Cooper test) perceived less fatigue, while maximal power (GS) did not differ between the three groups. Moreover, physical activity data accentuate the results found for physical performance, an increased Sport Index (physically active pupils) was found in LF compared with MF and HF. Even increased Leisure Time Index was found in the LF and MF groups compared with the HF group. Our results indicate that being physically active, even at a lower intensity, is related to lower SpF. Increased feelings of fatigue were found in adolescents with a physical disability (31) and in high school pupils $(24,33)$ with sedentary behaviors.

Interestingly, increased School/Work Index resulted in increased general fatigue and was linked to increased activity and motivation. Although these results should be interpreted with caution, it is possible that the assessed pupils (who work after school time in a bakery, a restaurant, etc.) are motivated and active in a work-related manner but experience increased general fatigue due to this work.

Knowing the negative impact of increased fatigue on cognitive functioning (25), it is necessary to stimulate physical activity and sports at schools in order to prevent the development of future obesity-related morbidities and to reduce fatigue in adolescents. Beside the physical aspects, schools should emphasize the importance of healthy habits in order to reduce increased fat mass.

Strengths of the study were the specific population (pupils with a technical and professional curriculum), large number of assessed pupils, and the fact that we included body composition parameters instead of BMI.

In this study, there are some limitations that have possible implications to data interpretation. For this study, we included pupils of one high school, this could implicate a possible selection bias, and future research should include multiple high schools. SpF was assessed using the questionnaire developed by Smets and colleagues (MFI-20). This questionnaire was not validated in obese adolescents, and using this questionnaire in the population of interest may be subject to measurement error. Although good internal consistency was found, results should be interpreted with caution. This study used a cross-sectional design, which precludes conclusions regarding causality.

\section{CONCLUSION}

An increased sense of fatigue was found in adolescents with increased fat percentage, reduced physical performance, and decreased physical activity. This study emphasises the importance of using fat mass and fat percentage instead of BMI when screening adolescents to determine their health status. To prevent increased fatigue, it is necessary to stimulate youngsters to be physically active and to promote healthy behaviors.

\section{ACKNOWLEDGMENTS}

This research was supported by APB Provinciaal Onderwijs Antwerpen, in particular the board of PIVA. We also thank the commitment of the department of Physical Educational of PIVA for data acquisition, logistics, and technical support.

\section{ETHICAL APPROVAL}

All procedures performed in studies involving human participants were in accordance with the ethical standards of the institutional and/or national research committee and with the 1964 Helsinki declaration and its later amendments or comparable ethical standards. For this type of study, formal consent is not required.

\section{Disclosure: The authors declare no conflict of interest.}

\section{REFERENCES}

1. deWater E, Braams BR, Crone EA, Peper JS. Pubertal maturation and sex steroids are related to alcohol use in adolescents. Horm Behav 2013;63: 392-7.

2. Wang YF. Is obesity associated with early sexual maturation? A comparison of the association in American boys versus girls. Pediatrics 2002;110:903-10.

3. Marcovecchio ML, Chiarelli F. Obesity and growth during childhood and puberty. World Rev Nutr Diet 2013;106:135-41.

4. Cheng G, Buyken AE, Shi L, et al. Beyond overweight: nutrition as an important lifestyle factor influencing timing of puberty. Nutr Rev 2012;70:133-52.

5. Pangrazi RP, Beighle A, Vehige T, Vack C. Impact of Promoting Lifestyle Activity for Youth (PLAY) on children's physical activity. J Sch Health 2003;73:317-21.

6. Strong WB, Malina RM, Blimkie CJ, et al. Evidence based physical activity for school-age youth. J Pediatr 2005;146:732-7. 


\section{Articles | Vantieghem et al.}

7. Tremblay MS, Leblanc AG, Janssen I, et al. Canadian sedentary behaviour guidelines for children and youth. Appl Physiol Nutr Metab 2011;36:59-71.

8. Type 2 diabetes in children and adolescents. American Diabetes Association. Pediatrics 2000;105 (Pt 1): 671-80.

9. Hansen HS, Froberg K, Hyldebrandt N, Nielsen JR. A controlled study of eight months of physical training and reduction of blood pressure in children: the Odense schoolchild study. BMJ 1991;303:682-5.

10. Mitchell JA, Pate RR, Dowda M, et al. A prospective study of sedentary behavior in a large cohort of youth. Med Sci Sports Exerc 2012;44:1081-7.

11. Brownson RC, Boehmer TK, Luke DA. Declining rates of physical activity in the United States: what are the contributors? Annu Rev Public Health 2005;26:421-43.

12. Toriola OO, Monyeki MA, Toriola AL. Two-year longitudinal healthrelated fitness, anthropometry and body composition status amongst adolescents in Tlokwe Municipality: the PAHL study. Afr J Prim Health Care Fam Med 2015;7:896.

13. Duvigneaud N, Matton L, Wijndaele K, et al. Relationship of obesity with physical activity, aerobic fitness and muscle strength in Flemish adults. J Sports Med Phys Fitness 2008;48:201-10.

14. HISIA Belgian Health Interview Survey - interactive analysis, 2011. https://www.wiv-isp.be/epidemio/hisia/index.htm.

15. Raitakari OT, Juonala M, Viikari JSA. Obesity in childhood and vascular changes in adulthood: insights into the cardiovascular risk in Young Finns Study. Int J Obesity 2005;29:S101-4.

16. Berenson GS, Srinivasan SR, Bao WH, et al. Association between multiple cardiovascular risk factors and atherosclerosis in children and young adults. N Engl J Med 1998;338:1650-6.

17. Morrison KM, Shin S, Tarnopolsky M, Taylor VH. Association of depression \& health related quality of life with body composition in children and youth with obesity. J Affect Disord 2014;172C:18-23.

18. Rank M, Wilks DC, Foley L, et al. Health-related quality of life and physical activity in children and adolescents 2 years after an inpatient weight-loss program. J Pediatr 2014;165:732-7 e2.

19. Varni JW, Burwinkle TM, Katz ER, Meeske K, Dickinson P. The PedsQL in pediatric cancer: reliability and validity of the Pediatric Quality of Life Inventory Generic Core Scales, Multidimensional Fatigue Scale, and Cancer Module. Cancer 2002;94:2090-106.

20. Varni JW, Burwinkle TM, Szer IS. The PedsQL Multidimensional Fatigue Scale in pediatric rheumatology: reliability and validity. J Rheumatol 2004;31:2494-500.
21. Varni JW, Burwinkle TM,, Limbers CA, Szer IS. The PedsQL as a patientreported outcome in children and adolescents with fibromyalgia: an analysis of OMERACT domains. Health Qual Life Outcomes 2007;5:9.

22. Palmer SN, Meeske KA, Katz ER, Burwinkle TM, Varni JW. The PedsQL Brain Tumor Module: initial reliability and validity. Pediatric Blood Cancer 2007;49:287-93.

23. Varni JW, Limbers CA, Bryant WP, Wilson DP. The PedsQL multidimensional fatigue scale in pediatric obesity: feasibility, reliability and validity. Int J Pediatr Obes 2010;5:34-42.

24. Viner RM, Clark C, Taylor SJ, et al. Longitudinal risk factors for persistent fatigue in adolescents. Arch Pediatr Adolesc Med 2008;162: 469-75.

25. Mizuno K, Tanaka M, Fukuda S, Imai-Matsumura K, Watanabe Y. Relationship between cognitive functions and prevalence of fatigue in elementary and junior high school students. Brain Dev 2011;33: 470-9.

26. Wan CS, Ward LC, Halim J, et al. Bioelectrical impedance analysis to estimate body composition, and change in adiposity, in overweight and obese adolescents: comparison with dual-energy X-ray absorptiometry. BMC Pediatr 2014;14:249.

27. Baecke JA, Burema J, Frijters JE. A short questionnaire for the measurement of habitual physical activity in epidemiological studies. Am J Clin Nutr 1982;36:936-42.

28. Smets EMA, Garssen B, Bonke B, Dehaes JCJM. The Multidimensional Fatigue Inventory (MFI) psychometric qualities of an instrument to assess fatigue. J Psychosom Res 1995;39:315-25.

29. Bautmans I, Mets T. A fatigue resistance test for elderly persons based on grip strength: reliability and comparison with healthy young subjects. Aging Clin Exp Res 2005;17:217-22.

30. Bautmans I, Onyema O, Van Puyvelde K, Pleck S, Mets T. Grip work estimation during sustained maximal contraction: validity and relationship with dependency and inflammation in elderly persons. J Nutr Health Aging 2011;15:731-6.

31. Maher C, Crettenden A, Evans K, et al. Fatigue is a major issue for children and adolescents with physical disabilities. Dev Med Child Neurol 2015;57:742-7.

32. Lim WJ, Hong S, Nelesen R, Dimsdale JE. The association fo obesity, cytokine levels, and depressive symptoms with diverse measures of fatigue in healthy subjects. Arch Intern Med 2005;165:910-5.

33. ter Wolbeek M, van Doornen LJ, Kavelaars A, Heijnen CJ. Severe fatigue in adolescents: a common phenomenon? Pediatrics 2006;117:e1078-86. 